\title{
Rettungswesen in der Schweiz - eine Bestandesaufnahme*
}

\author{
B. Zingg' ${ }^{a}$ C. Heim ${ }^{a}$, K. Meier ${ }^{a}$, D. Reinhardt ${ }^{b}$
}

\section{Zusammenfassung}

Eine Umfrage zu Strukturen und Einsatztätigkeit der bodengebundenen Rettungsdienste (RD) in der Schweiz über das Jahr 2001 zeigt, dass die RD je nach Trägerschaft und geographischer Region sehr unterschiedlich strukturiert sind. Bei Einsätzen mit potentieller Lebensbedrohung (D1) fährt in über 9 von 10 Fällen mindestens 1 diplomierter Rettungssanitäter mit. In über $80 \%$ der Fälle erreicht der Rettungsdienst innert 15 Minuten nach Alarmeingang den Notfallort. Notarztdienste sind kantonal und regional unterschiedlich organisiert. In dieser Funktion werden rund $3 / 5$ Ärzte (Anästhesisten und andere Fachärzte, zum Teil noch in Weiterbildung) und 2/5 Anästhesiepflegepersonen eingesetzt. Anästhesieabteilungen, welche sich am Rettungsdienst beteiligen, reduzieren dadurch teilweise ihre spitalinterne Notfallkapazität. Im Rahmen der knapperen Ressourcen muss die Entwicklung des schweizerischen Rettungswesens diskutiert werden, wozu diese Arbeit eine Grundlage liefern will.

a Anästhesie, Intensiv- und Notfallmedizin, Kantonsspital, Münsterlingen

b Institut für Anästhesiologie, Kantonales Spital, Uznach

* Diese Arbeit entstand aus der noch nicht eingereichten Dissertation von Frau B. Zingg. Die vorzeitige Publikation der wichtigsten Resultate erfolgt mit Genehmigung von Prof. Burg, Dekan der Medizinischen Fakultät der Universität Zürich. Die vollständige Datensammlung kann nach Genehmigung der Dissertation bei den Autoren angefordert werden.

Korrespondenz:

Dr. med. Christof Heim

Intensiv- und Notfallmedizin

Kantonsspital

CH-8596 Münsterlingen

Tel. 0716861111

Fax 0716862679

E-Mail: christof.heim@stgag.ch

\section{Einleitung}

Die zunehmende und zeitaufwendige notfallmedizinische Tätigkeit der Anästhesisten hat im Jahre 2001 den Vorstand der Schweizerischen Gesellschaft für Anästhesiologie und Reanimation SGAR bewogen, eine Kommission für Notfallmedizin $\mathrm{zu}$ bilden. Als erstes Projekt beschloss diese Kommission Anfang 2002, eine Bestandesaufnahme der Beteiligung der Anästhesieabteilungen im RD durchzuführen. Da es nicht möglich erschien, diese Fragestellung ohne Kenntnisse der Situation des gesamten Rettungswesens in der Schweiz zu beantworten, wurde der Umfang der Erhebung entsprechend erweitert. Dabei wurden materielle, personelle und finanzielle Aspekte untersucht. Die Erhebung erfolgte als selbständige Arbeit der Autoren mit Unterstützung von IVR, SGNOR und SMEDREC (Abkürzungen siehe Tab. 1).

\section{Material und Methode}

Mittels eines zweisprachigen Fragebogens (d/f) wurden die RD der ganzen Schweiz, die wir anhand einer IVR-Liste, über persönliche Kontakte und die SNZ identifizieren konnten, angeschrieben. Die Flugrettungsdienste sowie die reinen Krankentransportunternehmen wurden bei der Erhebung ausgeschlossen. Verschickt wurden die Fragebogen im März 2002, Endpunkt der Datenerhebung war nach teilweise bis zweimaliger Mahnung das Jahresende 2002. Die erhobenen Zahlen beziehen sich auf das Jahr 2001. Mangelhafte oder fehlende Informationen auf den zurückgeschickten Fragebogen wurden mittels telefonischer Nachfrage ergänzt. Dennoch bestehen kleine Lücken, meist wegen nicht greifbarer Daten oder laufender Umstrukturierungen.

Der Fragebogen war in die Kapitel Grunddaten, Struktur und Grösse des RD, Personal, Besatzung im Einsatz, Notarztdienst, Finanzen sowie Beteiligung der Anästhesieabteilungen gegliedert.

Die erhobenen Daten wurden der Betriebsstatistik der RD entnommen, bei fehlenden oder ungenauen Zahlen aber geschätzt. Einige Angaben konnten als Prozentbereiche geschätzt werden, welche dann mit Hilfe der Einsatzzahlen hochgerechnet wurden. Aus diesem Grund können wir aus den vorliegenden Daten keine wissenschaftlich korrekte Statistik errechnen, sondern nur Trends aufzeigen. Wegen dieser methodisch bedingten Ungenauigkeiten und zur besseren Visualisierung werden viele Resultate in graphischer Form dargestellt, ohne absolute Zahlen anzugeben.

Die Einzugsgebiete der einzelnen RD konnten nicht überall präzise abgegrenzt werden, insbesondere weil in einzelnen Regionen Überschneidungen vorkommen. Aus diesen Gründen werden Auswertungen mit Bezug auf die zu versorgende Population nur aufgrund der amtlichen Bevölkerungszahlen der Kantone vorgenommen. 
Tabelle 1

Abkürzungen und Internetadressen.

\begin{tabular}{|c|c|c|}
\hline BfS & Bundesamt für Statistik & www.statistik.admin.ch \\
\hline D1a & \multicolumn{2}{|c|}{$\begin{array}{l}\text { Notfall mit Beeinträchtigung der Vitalfunktionen, Ausfahrt mit Notarzt } \\
\text { gemäss Indikationenliste }\end{array}$} \\
\hline D1b & \multicolumn{2}{|l|}{ Notfall mit möglicher Beeinträchtigung der Vitalfunktionen } \\
\hline D2 & \multicolumn{2}{|l|}{ Notfall ohne vermutete Beeinträchtigung der Vitalfunktionen } \\
\hline D3 & \multicolumn{2}{|l|}{ Einsatz nach Vorbestellung } \\
\hline EA & \multicolumn{2}{|c|}{$\begin{array}{l}\text { Einsatzambulanz (Ausrüstung entspricht derjenigen eines Rettungswagens, } \\
\text { jedoch kleinerer Innenraum) }\end{array}$} \\
\hline IVR & Interverband für Rettungswesen & www.ivr.ch \\
\hline KTW & \multicolumn{2}{|l|}{ Krankentransportwagen } \\
\hline NA & \multicolumn{2}{|c|}{$\begin{array}{l}\text { Notarzt, Arzt mit spezieller Ausbildung in Wiederbelebung und anderen notfall- } \\
\text { medizinischen Massnahmen, Fähigkeitsausweis FMH seit dem 1. Januar } 2000\end{array}$} \\
\hline NEF & \multicolumn{2}{|c|}{$\begin{array}{l}\text { Notarzteinsatzfahrzeug (Personenwagen mit medizinischer Ausrüstung } \\
\text { zur notärztlichen Erstversorgung eines Patienten) }\end{array}$} \\
\hline RD & \multicolumn{2}{|l|}{ Rettungsdienst } \\
\hline RS & \multicolumn{2}{|l|}{ Rettungssanitäter } \\
\hline RTW & \multicolumn{2}{|l|}{ Rettungswagen } \\
\hline SDK & \multicolumn{2}{|c|}{$\begin{array}{l}\text { Schweizerische Konferenz der kantonalen Gesundheits- } \\
\text { direktoren }\end{array}$} \\
\hline SGAR / SSAR & \multicolumn{2}{|c|}{$\begin{array}{l}\text { Schweizerische Gesellschaft für Anästhesiologie und Reanimation / } \\
\text { Société Suisse d'anesthésiologie et réanimation } \quad \text { www.sgar-ssar.ch }\end{array}$} \\
\hline SGNOR & \multicolumn{2}{|l|}{$\begin{array}{l}\text { Schweizerische Gesellschaft für Notfall- und Rettungs- } \\
\text { medizin }\end{array}$} \\
\hline SMEDREC & \multicolumn{2}{|l|}{ Swiss Medical Resuscitation Council } \\
\hline SNZ & \multicolumn{2}{|l|}{ Sanitätsnotrufzentrale 144} \\
\hline VRS & Vereinigung Rettungssanitäter der Schweiz & www.vrs.ch \\
\hline
\end{tabular}

Abbildung 1

Geographische Verteilung der Antworten.

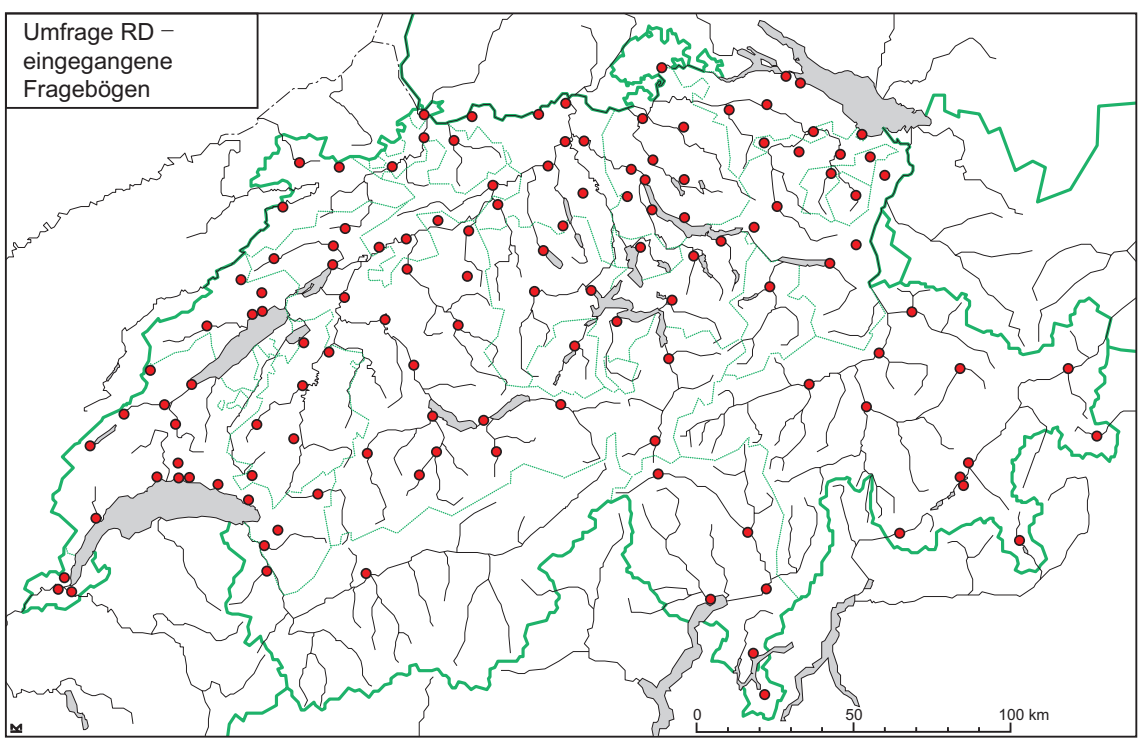

Um Faktoren zu eruieren, die die heutige Versorgung beeinflussen können, wurden die Rettungsdienste bei der Auswertung nach verschiedenen Gesichtspunkten gruppiert: Kanton; Topographie; Sprache; Trägerschaft; Grösse des RD; Funktion und Grösse des Spitals, falls RD eingebunden. Diese Unterteilung wurde von den Autoren empirisch, teilweise in Anlehnung an frühere Erhebungen, gemacht.

\section{Resultate}

Von den 157 angeschriebenen RD der Schweiz antworteten 128, was einer Rücklaufquote von $82 \%$ entspricht (Abb.1). Bei der Auswertung rechneten wir mit 121 Datensätzen, da 7 früher eigenständige RD als Nebenstandorte eines grösseren RD betrieben werden und ihre Zahlen in der Antwort des letzteren integriert sind. Dank der zweisprachigen Abfassung des Fragebogens entstanden keine grossen Unterschiede zwischen den Sprachgebieten.

Von den $121 \mathrm{RD}$ sind 67 (55 \%) an öffentliche Spitäler gebunden, 36 (30\%) sind selbständige RD mit öffentlicher Trägerschaft und 18 (15\%) private $\mathrm{RD}$, wovon $2 / 3$ mit Leistungsauftrag der öffentlichen Hand.

Die erfassten Rettungsdienste verfügen nebst Spezialfahrzeugen (Einsatzleitung, Katastrophenfahrzeug, Snowmobile) über 453 Fahrzeuge, davon 74 (16\%) KTW, 133 (29\%) EA, 203 (45\%) RTW und 43 (10\%) NEF (Abb. 2). Einsatzambulanzen sind in den Gebirgs- und Voralpenregionen weiter verbreitet als in den städtischen Gebieten. Die Aufteilung nach Kantonen zeigt, dass die Ballungsgebiete mit relativ weniger Einsatzmitteln auskommen.

Von den 453 Einsatzmitteln können unter optimalen Bedingungen (= werktags) mit dem regulär zum Dienst eingeteilten Personal 274 Fahrzeuge betrieben werden, im schwierigsten Zeitpunkt (= Wochenende, nachts) 189. Von diesen 189 Fahrzeugen ist bei 111 (59\%) die Besatzung am Standort des RD, bei 78 (41\%) befindet sich die Besatzung in Pikettstellung zu Hause.

Die befragten RD leisteten im Jahr 2001 277000 Einsätze, wovon 103557 (38 \%) als D1 erfolgten, d.h. Einsätze mit manifester oder vermuteter Beeinträchtigung der Vitalfunktionen (Abb. 3). Aufgeteilt nach topographischen Regionen zeigt sich, dass vor allem die ländlichen und alpinen RD kleinere Einsatzzahlen haben. Während in den grossem Ballungszentren die RD durchschnittlich 9936 Einsätze pro Jahr leisten, sind es in den mittleren Städten 3500, im Mit- 
Abbildung 2

Rettungsmittel / 100000 Einwohner.

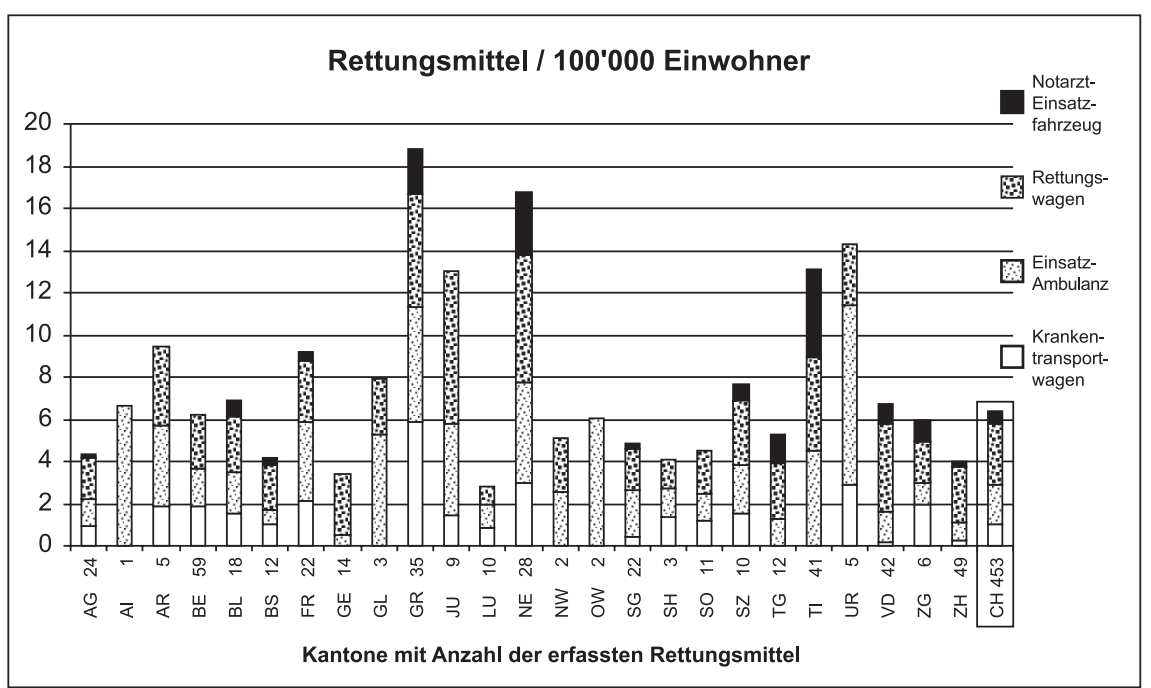

Abbildung 3

Einsätze / 1000 Einwohner nach Kantonen.

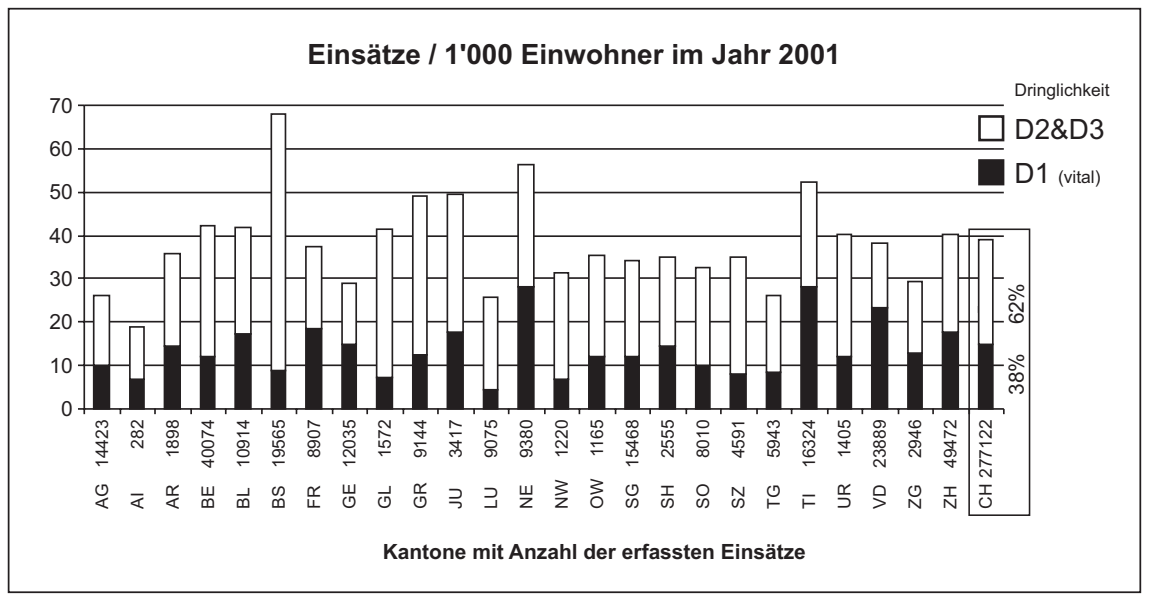

Abbildung 4

Anteil Vollzeit-/Milizarbeit nach Kanton.

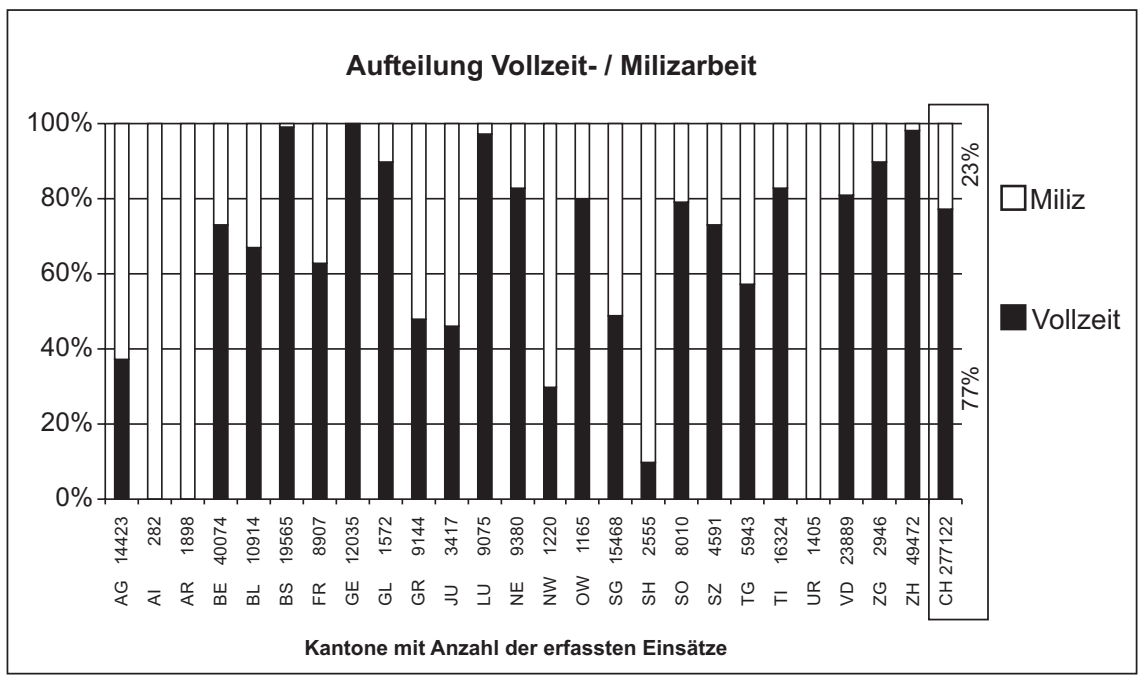

telland 1734 und in den Alpen 669 pro RD. Die Einsätze pro Fahrzeug bewegen sich für die einzelnen RD zwischen 28 und 2130 pro Jahr, im Durchschnitt der Regionen zwischen 257 (Alpen) und 1152 (Städte).

Beim Personal wurde einerseits nach Vollzeitund Milizarbeit sowie andererseits nach den Berufsgruppen gefragt. Als Vollzeitmitarbeiter wurden diejenigen definiert, welche zu über $80 \%$ ihrer Arbeitszeit für den Rettungsdienst arbeiten, die anderen als Miliz (Abb. 4). Diese Einteilung nimmt keinen Bezug auf die berufliche Qualifikation der einzelnen Personen. Die einzelnen RD schätzten den Arbeitsanteil der beiden Gruppen in Prozentwerten. Vor allem die kleineren Rettungsdienste in den kleineren öffentlichen Spitälern der Grundversorgung sind im Milizsystem organisiert, d. h. das Personal wird ausserhalb der effektiven Einsatzzeit in unterschiedlichem Ausmass für andere Aufgaben im Spital eingesetzt. Hochgerechnet mit den Einsatzzahlen werden 3/4 der Arbeit durch Vollzeitund $1 / 4$ durch Milizmitarbeiter geleistet.

Die befragten RD haben 2804 Mitarbeiter eingestellt, wovon 1016 diplomierte RS, 206 RS in Ausbildung und 307 Pflegefachpersonen sind. Bei den restlichen 1275 Personen handelt es sich um Transporthelfer, Samariter sowie vereinzelte Angestellte in Administration und Technik. Nach dem «technicien ambulancier» (Gehilfe des RS mit verkürzter Ausbildung, momentan vor allem in der französischen Schweiz im Einsatz) wurde nicht gezielt gefragt. Oft handelt es sich um Arbeitsverhältnisse in Teilzeit, und die vorliegenden Daten erlauben keine Aussage über den Anteil der einzelnen Berufsgruppen an der effektiven Einsatztätigkeit.

Als Hilfsfrist wird die Zeitspanne vom Alarmeingang beim RD bis zum Eintreffen am Ereignisort definiert. Sie sollte gemäss den Richtlinien des IVR für $90 \%$ der D1-Einsätze in städtischen Gebieten maximal 10, in ländlichen Regionen maximal 15 Minuten betragen. Wir haben nur nach der 15-Minuten-Limite gefragt. Die Erhebung dieser Zeiten erfolgt teilweise mittels elektronisch übermittelter Statusmeldungen und automatischer Speicherung im Einsatzleitrechner, teilweise aber manuell. Die RD gaben daher einen (gemessenen oder geschätzten) Prozentbereich an, in welchem sie das Kriterium erfüllten. Hochgerechnet mit den Einsatzzahlen erhielten wir gesamtschweizerisch einen Wert für die Erfüllung der 15-Minuten-Limite von $89 \%$, schwankend zwischen $82 \%$ in alpinen und $94 \%$ in städtischen Regionen (Tab. 2). Zudem wird die Hilfsfrist von 15 Minuten in der Romandie und im Tessin zu $93 \%$ respektive $94 \%$ 
eingehalten, in der deutschen und rätoromanischen Schweiz in $86 \%$. Die RD konnten Gründe für verlängerte Hilfsfristen angeben: Oft wurde die Distanz genannt, dann mit abnehmender Häufigkeit die Ausrückzeit (Pikettstellung zu Hause), der Verkehr (Berufs- und Ferienverkehr) sowie andere Ursachen wie z. B. Fahrten in andere Einsatzgebiete.

Tabelle 2

Einhaltung der Hilfsfrist von 15 Minuten.

\begin{tabular}{lr} 
Anzahl antwortende RD & 114 \\
\hline Einsätze total & 265244 \\
\hline Einsätze D1 & 101221 \\
\hline $\begin{array}{l}\text { davon Hilfsfrist 15' eingehalten } \\
\text { (Hochrechnung) }\end{array}$ & 89874 \\
\hline Anteil & $89 \%$
\end{tabular}

Abbildung 5

Rettungssanitäter bei D1-Einsatz nach Kanton.

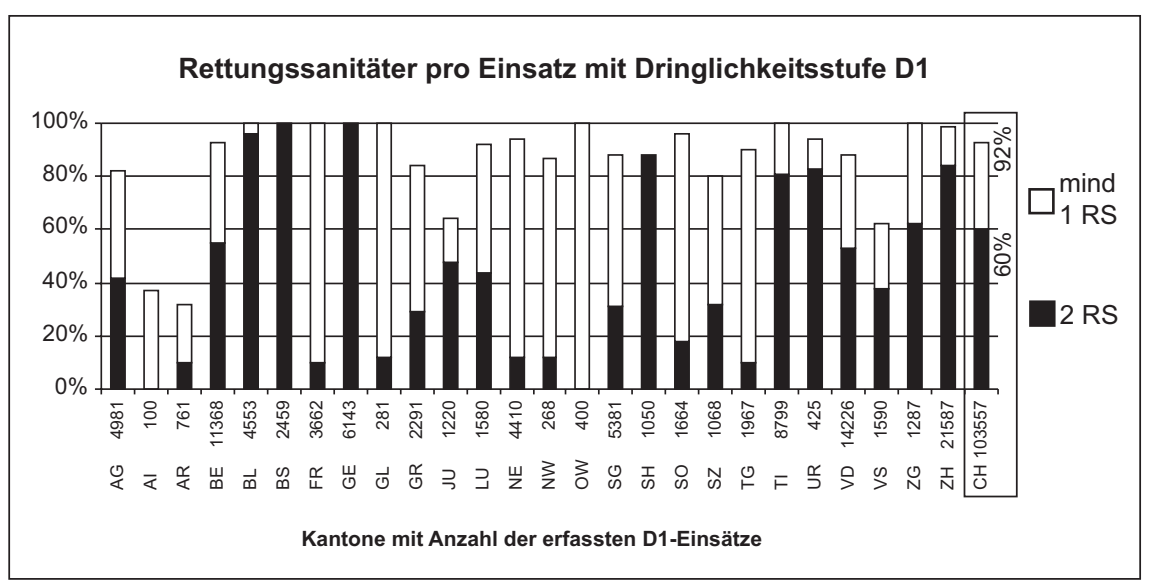

Abbildung 6

D1-Einsätze mit/ohne NA pro 1000 Einwohner.

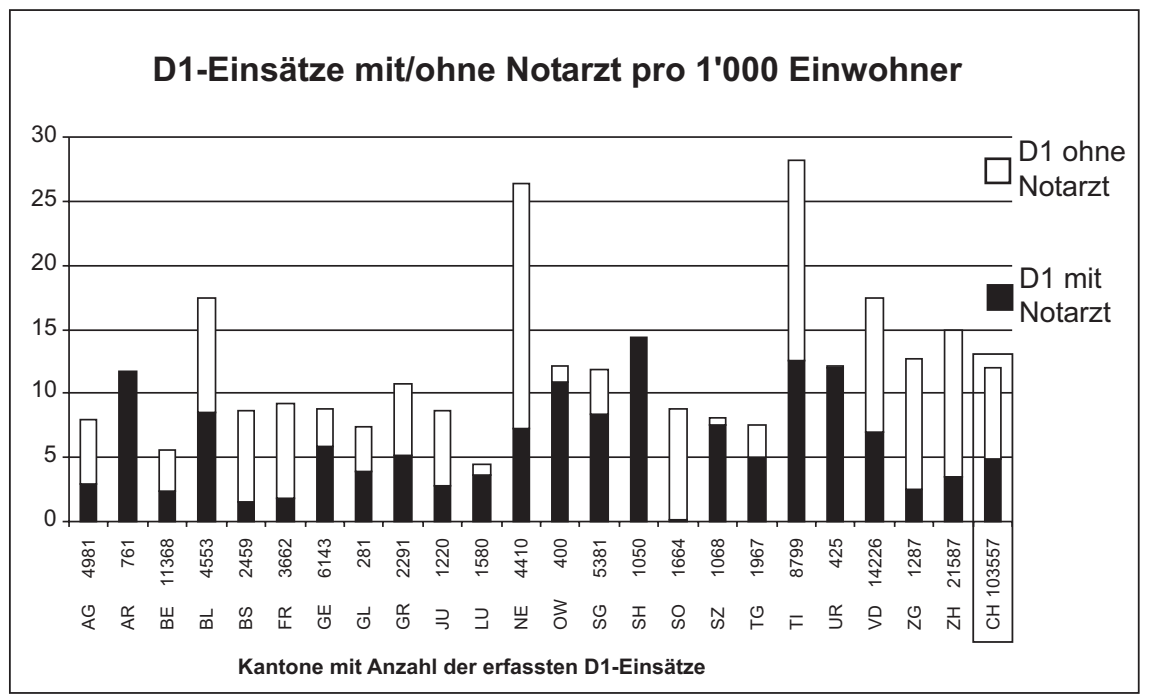

Bezüglich des Einsatzes von Rettungssanitätern wurde erfragt, bei welchem Prozentsatz von D1-Einsätzen die einzelnen RD zwei oder mindestens einen diplomierten RS einsetzen können. Hochgerechnet mit den Einsatzzahlen finden wir, dass in $60 \%$ der D1-Einsätze zwei RS und in weiteren $32 \%$ (= total in 92\%) mindestens 1 RS ausfahren. Auch hier zeigt sich der Einfluss von Grösse des RD, der Topographie und eine sehr unterschiedliche kantonale Verteilung (Abb.5). Wer bei den restlichen Einsätzen $(\mathrm{CH}=8 \%)$ ausfährt, geht aus unseren Daten nicht hervor.

Bei den Notarzteinsätzen zeigt es sich, dass die einzelnen Kantone einen unterschiedlich hohen Anteil haben (Abb. 6).

Wer kommt als NA zum Einsatz? Die RD konnten einen Prozentanteil angeben, welcher mit den Zahlen der NA-Einsätze hochgerechnet wurde (Tab.3). Die Kantone haben unterschiedliche NA-Strukturen und schicken entsprechend unterschiedliche Personalgruppen in diese Einsätze. Teilweise kommt die Anästhesiepflege als Ersatz für den NA zum Einsatz, vorwiegend im spitalgebundenen RD in ländlichen Gebieten (Abb. 7).

Tabelle 3

Notarzteinsätze.

\begin{tabular}{lr} 
Anzahl antwortende RD & 94 \\
\hline Notarzteinsätze total & 38513 \\
\hline Durch Anästhesieärzte & $27 \%$ \\
\hline Durch andere Ärzte & $32 \%$ \\
\hline Durch Anästhesiepflege & $41 \%$
\end{tabular}

Die Beteiligung einer Anästhesieabteilung wurde bei 69 RD erwähnt und näher bezeichnet (Tab. 4).

Tabelle 4

Beteiligung der Anästhesieabteilungen am Rettungsdienst.

\begin{tabular}{lc}
\hline Antwort & Nennungen \\
\hline Gesamtleitung & 26 \\
\hline Ärztliche Leitung & 54 \\
\hline Einsatz von Anästhesiepflegepersonal & 19 \\
\hline In RS-Funktion routinemässig & 23 \\
\hline Als Arztersatz routinemässig & 30 \\
\hline Als Arztersatz in Ausnahmefällen & 14 \\
\hline Einsatz von Anästhesieärzten & 30 \\
\hline Anderes & 7 \\
\hline Zahl der antwortenden RD & 69 \\
\hline (Mehrfachnennungen möglich) &
\end{tabular}

61 Abteilungen nahmen auch Stellung zum Einfluss der RD-Einsätze auf ihre spitalinterne 
Abbildung 7

Wer fährt die Notarzteinsätze?

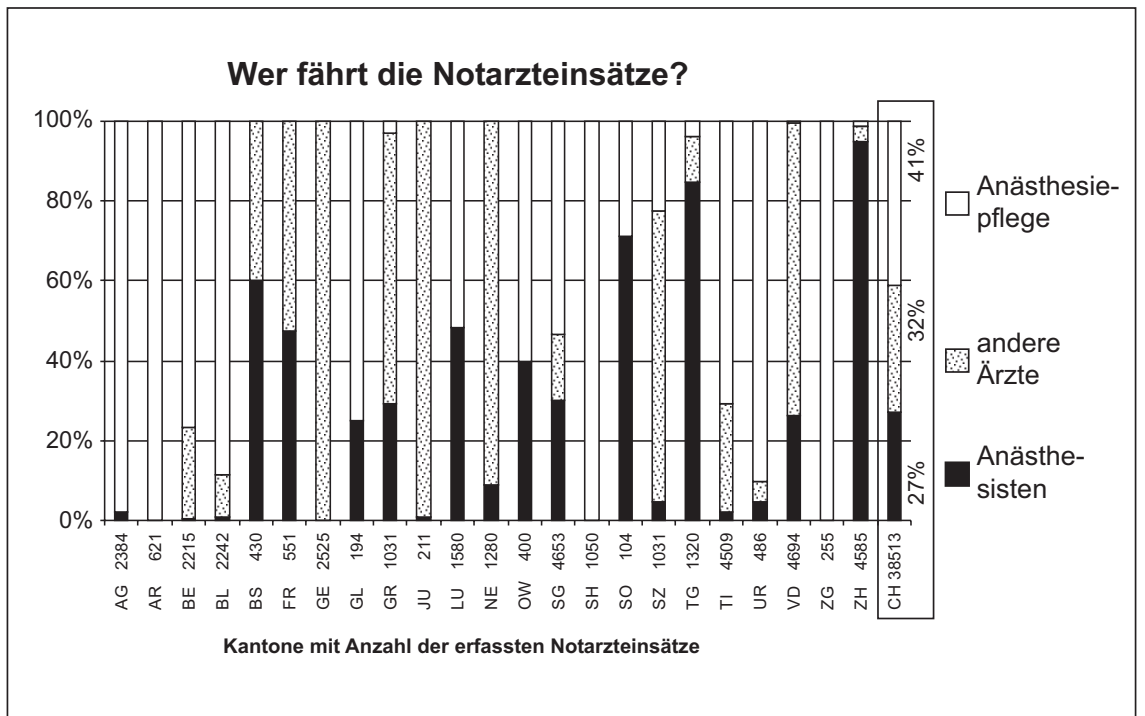

Tätigkeit. 17mal wurde angegeben, dass eine Operationstätigkeit durch eine separate Equipe jederzeit gewährleistet ist. An 21 Häusern ist eine zweite Equipe zu Hause in Pikettstellung, und an 13 Spitälern müsste für eine gleichzeitige Anästhesie Personal in der Freizeit mobilisiert werden. 13mal wurden andere Varianten genannt, wovon 10mal, dass eine allfällige Anästhesie durch eine Person alleine durchgeführt würde.

88 Rettungsdienste beantworteten die Frage nach ihren Finanzquellen. Wiederum über die Einsatzzahlen hochgerechnet ergibt sich, dass gut $60 \%$ der Kosten durch Verrechnung an Patient oder Versicherung gedeckt sind. Weitere 10\% können intern verrechnet werden, d. h., es sind z. B. Transporte von KVG-Patienten, welche zu Lasten des Spitals gehen (Verlegungen, auswärtige Diagnostik). Gut 20\% des Aufwandes sind budgetiert, hauptsächlich durch die Trägerschaft gedeckte Vorhaltekosten. Defizitgarantien und andere Quellen machen nur etwa $5 \%$ aus.

\section{Diskussion}

Die Bestandesaufnahme gibt einen aktuellen Überblick über das Rettungswesen der Schweiz.

Für die vielfältigen Organisationsformen sind die kantonalen Gesetze, die Finanzierungsweise sowie die lokalen Spitalstrukturen verantwortlich. An einigen Orten sind zudem grossflächige Restrukturierungsprozesse im Gange (z. B. Genf, Freiburg-Süd), deren Abschluss das vorliegende Bild bereits wieder ändern wird.
Aus diesen Gründen können RD nur mit Einschränkungen und innert einer kurzen Zeitspanne beurteilt und verglichen werden.

Bei den Grunddaten wurde nach dem Einzugsgebiet des Rettungsdienstes gefragt. Es musste jedoch relativ rasch festgestellt werden, dass sich aus den folgenden Gründen nur eine eingeschränkte Beziehung zu den übrigen Kenndaten der RD machen liess:

Die Einzugsgebiete einzelner RD können sich überschneiden, wenn auch mit verschiedenen Funktionen. So gaben zum Beispiel die Thurgauer RD an, eine Summe von total 305000 Einwohner zu versorgen, der Kanton hat aber nach BfS nur 228000 Einwohner.

Aus diesem Grunde wurden bei der Auswertung nach Kantonen Beziehungen zur Bevölkerungszahl (BfS) gemacht, wobei der Kanton Wallis wegen lediglich 2 Antworten ausgelassen wurde. Diese Resultate sind jedoch vorsichtig zu interpretieren: Kantone wie BS, GE und TI haben ausserordentlich hohe Anteile an Grenzgängern mit grösseren Populationen an Werktagen. Eine ähnliche Problematik kennen die Tourismuskantone.

Die Häufigkeit von D1-Einsätzen ist in den Kantonen sowie in den Sprachregionen sehr unterschiedlich. Die Frage nach der Ursache beantworten unsere Daten nicht. Mögliche Gründe sind unterschiedliche Mentalitäten, Indikationenlisten und Taktiken der SNZ, Vergabe von D3-Einsätzen an Krankentransportunternehmen (in unseren Zahlen nicht berücksichtigt), verschiedene Sicherheitsbedürfnisse der Disponenten, unterschiedliche Basismittel und Personalreserven.

Die Fahrzeugdichte variiert stark. In der Stadt kann in bezug auf Hilfsmittel effizienter gearbeitet werden, da sich die zu versorgenden Personen auf kleinerem Raum aufhalten. Die Nutzung der einzelnen Fahrzeuge schwankt enorm. Dies wirkt sich auf die Fixkosten aus, da die Amortisation der Fahrzeuge einen wesentlichen Teil der Materialkosten ausmacht. Beim Typ des Fahrzeuges zeigt sich in den (vor-)alpinen Regionen ein Trend zur Anschaffung von Einsatzambulanzen anstelle von RTW. Diese sind gleich ausgerüstet, aber kleiner und deshalb in engen Verhältnissen manövrierfähiger.

Notarzteinsatzfahrzeuge sind teilweise dicht positioniert. Dies widerspiegelt die unterschiedlichen kantonalen sowie regionalen Strukturen und vielleicht die zunehmende Zentralisierung der NA-Systeme. Die getrennte Zuführung von Rettungswagen und NA im sogenannten Rendezvous-System kann die Verfügbarkeit des Notarztes verbessern, bedingt aber in der Regel 
den zusätzlichen Aufwand eines RS als Fahrer. Der NA als Lenker des NEF ist aus einsatz- und sicherheitstechnischen Gründen umstritten.

Profi- und Milizsysteme werden, zum Teil mit fliessenden Übergängen, in den schweizerischen RD nebeneinander betrieben. Soll eine einheitliche, professionelle Strukturierung der Rettungsdienste gefordert werden? Gerade in ländlichen Regionen scheint das besonders nächtliche Pikettsystem (Miliz) ein optimales KostenNutzen-Verhältnis aufzuweisen. Ein qualitativer Einfluss auf die Grundversorgung kann mit unseren Daten nicht nachgewiesen werden. Rund 40\% der Besatzungen leistet nachts Pikett von zu Hause, was billiger ist als eine dauernde Anwesenheit am Standort des RD.

Oft werden RD-Standorte durch topographische Gegebenheiten erzwungen und bedingen damit suboptimale Grössen, wie die Aufschlüsselung der durchschnittlichen Einsatzzahlen pro RD nach geographischer Region zeigt. Ebenso kann keine allgemeingültige Schwelle für einen Übergang vom Miliz- zum Profisystem gefunden werden. In Gebieten geringer Rettungsdienstaktivität existieren Miliz- oder Mischsysteme, in dem neben Rettungssanitätern zum Beispiel auch speziell geschulte Notfall- oder Intensivpflegepersonen ausfahren.

Die Hilfsfrist ist eines der Mittel zur Beurteilung der Prozessqualität der rettungsdienstlichen Versorgung der Bevölkerung. Eine interne Qualitätskontrolle der Autoren in den Jahren 1997/98 ergab in einem RD im Mittelland, dass die 15-Minuten-Limite in 65\% eingehalten werden konnte. Zwei RD der Gruppe Stadt und Mittelland, welche ihre Zeiten elektronisch erfassen, gaben in unserer Umfrage ebenfalls einen Bereich von 60 bis $79 \%$ an. Aufgrund dieser Daten und aufgrund der geographischen Voraussetzungen der Schweiz müssen die Daten bezüglich Hilfsfristen hinterfragt werden. Eine Klärung wird erst durch die einheitliche Datenerhebung und die konsequente Verwendung von automatischen und elektronisch registrierten Statusmeldungen möglich werden. Die an öffentliche Spitäler gebundenen Rettungsdienste halten die Hilfsfrist nur in 83\% ein. Das hier häufige Milizsystem könnte die Ausrückzeit verzögern.

Beim Einsatz von Rettungssanitätern konnte ein beachtlicher Erfolg gefunden werden, welcher vor wenigen Jahren noch unrealisierbar schien: Bei D1-Einsätzen (mit oder ohne Arztbegleitung) fährt in durchschnittlich $92 \%$ mindestens 1 diplomierter RS aus. Soll die flächendeckende Forderung nach 2 Rettungssanitätern bei D1-Einsätzen aufrechterhalten werden? Klei- nere Rettungsdienste können es sich selten leisten, rund um die Uhr zwei RS vorzuhalten.

Aus der Umfrage geht nicht hervor, was für Personal bei den restlichen $8 \%$ der D1-Einsätze ausrückt. Es wird angenommen, dass bei spitalgebundenen RD ein grösserer Teil des diplomierten Pflegepersonals ohne RS-Ausbildung ist.

Bei den NA-Einsätzen besteht das Problem, dass verschiedene Begriffe vermischt werden:

- Als sogenannte «Notarzteinsätze» sind die D1a-Einsätze definiert: Notfall mit Beeinträchtigung der Vitalfunktionen (NA-Indikationenliste der FMH). Die Besatzung besteht in der Regel aus einem (Not-)Arzt sowie einer weiteren (bei NEF) bis zwei (bei RTW) weiteren Personen, davon mindestens ein diplomierter RS (Richtlinien über die Anerkennung von Rettungsdiensten IVR 2000);

- andererseits gibt es seit dem 1. Januar 2000 den Fähigkeitsausweis FMH «Notarzt».

Gemäss Umfrage sind verschiedene Berufsgruppen in der NA-Funktion tätig: FMH-Notärzte verschiedener Fachrichtungen, Notärzte in Weiterbildung, Ärzte ohne NA-Curriculum und Anästhesiepflegepersonal. Der Anteil des Pflegepersonals ist mit $41 \%$ unerwartet hoch. Unsere Daten erlauben leider keine Aussage darüber, wie viele der «NA-Einsätze» effektiv von Trägern des Fähigkeitsausweises Notarzt geleistet wurden.

Eine Anästhesieabteilung ist bei über 50\% der RD (69 von 121 Antworten) in der präklinischen Notfallmedizin involviert! Die Beteiligung betrifft hauptsächlich die ärztliche oder gesamtheitliche RD-Leitung und den direkten Einsatz von ärztlichem und pflegerischen Personal. In französisch- und italienischsprachigen Gebieten spielt diese Verknüpfung eine geringe Rolle.

Kritisch sind die Angaben über Beeinträchtigungen des Operationsbetriebes durch den Einsatz von Anästhesiepersonal im Rettungsdienst zu werten. Dass notfalls wegen eines RD-Einsatzes die Anästhesieführung durch eine einzelne Person erfolgt oder erfolgen müsste, widerspricht den SGAR-Standards.

\section{Schlussfolgerungen}

Unsere Daten weisen darauf hin, dass das bodengebundene Rettungswesen in der Schweiz in qualitativer und ökonomischer Hinsicht optimierbar ist. Die zu treffenden Massnahmen sind jedoch stark von der politischen Haltung der Beteiligten und Verantwortlichen abhängig. Siehe auch den politischen Kommentar zu dieser Arbeit auf Seite 1787-9. 